\title{
RAMANUJAN: A TALE OF TWO EVALUATIONS
}

\author{
DONALD J. MANZOLI
}

\begin{abstract}
In 1887, beneath a canopy of stars, Srinivasa Ramanujan commenced his brief existence on this planet. In a universe at the mercy of its entropy, against all odds, a genius was born. His destiny was mathematics, a subject born thousands of years earlier. The power of this discipline is not to be denied. After all, with our minds in the stars, we have placed footprints on the moon. The conquest of the moon was a triumph of applied mathematics; however, it was the landscape of pure mathematics that awaited Ramanujan. In time, he would explore it with passion, leaving footprints lasting for eternity.

Professor Bruce C. Berndt has done a remarkable job of editing the notebooks Ramanujan left behind. In particular, Berndt's Chapter 9 of Ramanujan's notebooks Part I provides a magnificent in-depth look at raw mathematical talent in action. The primary purpose of this article is to present three Chapter 9 related results, a series evaluation and two new functional equations, that Ramanujan either missed or his work on them was lost. The secondary purpose is to present what Berndt calls a "corrected version" [5, page 233] of an incorrect Chapter 9 formula of Ramanujan. This series evaluation represents one of the few serious mistakes to be found in Ramanujan's work.
\end{abstract}

1. A missing functional equation. This mathematical pioneer had a marvelous way of looking beyond the familiar, using his intuition to guide him through what, in his mind, was uncharted mathematical territory. For example, consider the following well-known power series:

$$
f(x)=\sum_{k=1}^{\infty} \frac{H_{k} x^{k+1}}{k+1}=\frac{1}{2} \ln ^{2}(1-x),
$$

where

$$
H_{k}=\sum_{j=1}^{k} \frac{1}{j} \quad \text { and } \quad-1 \leq x<1 .
$$

2010 AMS Mathematics subject classification. Primary 40 G99.

Keywords and phrases. Ramanujan, polylogarithms.

Received by the editors on August 8, 2012, and in revised form on May 21, 2014. 
For $x=1 / 2$, this yields

$$
f\left(\frac{1}{2}\right)=\sum_{k=1}^{\infty} \frac{H_{k}}{(k+1) 2^{k+1}}=\frac{1}{2} \ln ^{2} 2 .
$$

Ramanujan considered two analogues of $f(x)$, namely,

$$
g(x)=\sum_{k=1}^{\infty} \frac{H_{k} x^{k+1}}{(k+1)^{2}}, \quad h(x)=\sum_{k=1}^{\infty} \frac{H_{k} x^{k+1}}{(k+1)^{3}},
$$

where $-1 \leq x \leq 1$ [5, pages 251, 253]. (For simplicity in what follows, we will consider only real arguments.) Ramanujan's entry 9 (i) [5, page 251], [9, page 303, (12)] provides a formula, valid for $0<x<1$, that allows $g(1 / 2)$ to be evaluated.

$$
g(1-x)=\frac{1}{2} \ln ^{2} x \ln (1-x)+\mathrm{Li}_{2}(x) \ln x+\zeta(3)-\mathrm{Li}_{3}(x),
$$

where

$$
\operatorname{Li}_{n}(x)=\sum_{k=1}^{\infty} \frac{x^{k}}{k^{n}}, \quad \text { for } n>1 \text { and }-1 \leq x \leq 1,
$$

denotes the $n$th polylogarithm function [5, page 246]. Equivalently,

$$
\begin{aligned}
g(1-x)= & -\frac{1}{2} \ln ^{2} x \ln (1-x)+\zeta(2) \ln x-\operatorname{Li}_{2}(1-x) \ln x \\
& +\zeta(3)-\operatorname{Li}_{3}(x) .
\end{aligned}
$$

Since $\operatorname{Li}_{2}(1 / 2)=-(1 / 2) \ln ^{2} 2+(1 / 2) \zeta(2)$ and $\operatorname{Li}_{3}(1 / 2)=(1 / 6) \ln ^{3} 2-$ $(1 / 2) \zeta(2) \ln 2+(7 / 8) \zeta(3)$, we have

$$
g\left(\frac{1}{2}\right)=\sum_{k=1}^{\infty} \frac{H_{k}}{(k+1)^{2} 2^{k+1}}=-\frac{1}{6} \ln ^{3} 2+\frac{1}{8} \zeta(3) .
$$

Having evaluated $f(1 / 2)$ and $g(1 / 2)$, it is reasonable to investigate the possible evaluation of

$$
h\left(\frac{1}{2}\right)=\sum_{k=1}^{\infty} \frac{H_{k}}{(k+1)^{3} 2^{k+1}} .
$$

Although Ramanujan developed functional equations for $g(1-x)-$ $g(1-(1 / x))$ and $h(1-x)-h(1-(1 / x))$ (entry 9 (ii) [5, page 251] and entry 10 (i) [5, page 253], respectively), he evidently did not establish 
a formula for $h(1-x)$ alone, analogous to (1.1) for $g(1-x)$ (entry 9 (i)). Lewin [9] does not list such a result for $h(1-x)$ either. The "missing" formula, developed by the author, is

$$
\begin{aligned}
h(1-x)= & \frac{1}{24} \ln ^{4} x-\frac{1}{6} \ln ^{3} x \ln (1-x)+\frac{1}{2} \zeta(2) \ln ^{2} x \\
& +\zeta(3) \ln x-\operatorname{Li}_{3}(1-x) \ln x+\zeta(4) \\
& -\operatorname{Li}_{4}(x)+\operatorname{Li}_{4}(1-x)+\operatorname{Li}_{4}\left(1-\frac{1}{x}\right),
\end{aligned}
$$

where $1 / 2 \leq x<1$. For $x=1 / 2$, this yields

$$
h\left(\frac{1}{2}\right)=\sum_{k=1}^{\infty} \frac{H_{k}}{(k+1)^{3} 2^{k+1}}=\frac{1}{24} \ln ^{4} 2-\frac{1}{8} \zeta(3) \ln 2+\frac{1}{8} \zeta(4) .
$$

Note the similarity of (1.3) and (1.2), which is obtained from (1.1) by using a functional equation for the dilogarithm attributed to Euler (Ramanujan's entry 6 (iii) [5, page 247]).

$$
\mathrm{Li}_{2}(x)+\mathrm{Li}_{2}(1-x)=\zeta(2)-\ln x \ln (1-x),
$$

where $0<x<1$. Note also the cancellation of $\operatorname{Li}_{4}(1 / 2)$ in the evaluation of $h(1 / 2)$. Although $\operatorname{Li}_{2}(1 / 2)=-(1 / 2) \ln ^{2} 2+(1 / 2) \zeta(2)$ and $\operatorname{Li}_{3}(1 / 2)=(1 / 6) \ln ^{3} 2-(1 / 2) \zeta(2) \ln 2+(7 / 8) \zeta(3)$, a similar expression for $\operatorname{Li}_{4}(1 / 2)$ is not known [10, page 2].

The proof of (1.3) requires bringing in one additional formula, which is due to Landen (Ramanujan's entry 7 (i) [5, page 249]).

$$
\begin{aligned}
& \mathrm{Li}_{3}(1-x)+\mathrm{Li}_{3}\left(1-\frac{1}{x}\right)+\mathrm{Li}_{3}(x) \\
& =\frac{1}{6} \ln ^{3} x-\frac{1}{2} \ln ^{2} x \ln (1-x)+\zeta(2) \ln x+\zeta(3),
\end{aligned}
$$

where $1 / 2 \leq x<1$. Solving (1.2) for $-\mathrm{Li}_{3}(x)-\mathrm{Li}_{2}(1-x) \ln x$ and adding it to (1.6) gives us a new functional equation for the trilogarithm.

$$
\mathrm{Li}_{3}(1-x)+\mathrm{Li}_{3}\left(1-\frac{1}{x}\right)-\mathrm{Li}_{2}(1-x) \ln x=\frac{1}{6} \ln ^{3} x+g(1-x),
$$

where $1 / 2 \leq x<1$. (This can be derived directly for $1 / 2 \leq x \leq 2$.) The proof of (1.3) involves creating applications of (1.6) and (1.7). For 
$1 / 2 \leq x<1$, we have

$$
\begin{aligned}
& \left(\operatorname{Li}_{4}(1-x)\right)^{\prime}+\left(\operatorname{Li}_{4}\left(1-\frac{1}{x}\right)\right)^{\prime}-\left(\operatorname{Li}_{4}(x)\right)^{\prime}-\left(\operatorname{Li}_{3}(1-x) \ln x\right)^{\prime} \\
& =\left(\sum_{k=1}^{\infty} \frac{(1-x)^{k}}{k^{4}}\right)^{\prime}+\left(\sum_{k=1}^{\infty} \frac{(1-(1 / x))^{k}}{k^{4}}\right)^{\prime} \\
& -\left(\sum_{k=1}^{\infty} \frac{x^{k}}{k^{4}}\right)^{\prime}-\left(\ln x \sum_{k=1}^{\infty} \frac{(1-x)^{k}}{k^{3}}\right)^{\prime} \\
& =\frac{-1}{1-x} \operatorname{Li}_{3}(1-x)+\frac{1}{x^{2}} \frac{1}{1-(1 / x)} \mathrm{Li}_{3}\left(1-\frac{1}{x}\right)-\frac{1}{x} \mathrm{Li}_{3}(x) \\
& -\frac{1}{x} \operatorname{Li}_{3}(1-x)-\frac{-1}{1-x} \operatorname{Li}_{2}(1-x) \ln x \\
& =\frac{-1}{1-x}\left(\operatorname{Li}_{3}(1-x)+\operatorname{Li}_{3}\left(1-\frac{1}{x}\right)-\operatorname{Li}_{2}(1-x) \ln x\right) \\
& +\frac{-1}{x}\left(\operatorname{Li}_{3}(1-x)+\operatorname{Li}_{3}\left(1-\frac{1}{x}\right)+\operatorname{Li}_{3}(x)\right) \\
& =\frac{-1}{1-x}\left(\frac{1}{6} \ln ^{3} x+g(1-x)\right) \\
& +\frac{-1}{x}\left(\frac{1}{6} \ln ^{3} x-\frac{1}{2} \ln ^{2} x \ln (1-x)+\zeta(2) \ln x+\zeta(3)\right) \\
& =-\frac{1}{6} \frac{\ln ^{3} x}{x}+\frac{1}{2} \frac{\ln ^{2} x}{x} \ln (1-x)-\frac{1}{6} \frac{\ln ^{3} x}{1-x} \\
& -\zeta(2) \frac{\ln x}{x}-\frac{\zeta(3)}{x}-\frac{g(1-x)}{1-x} \text {. }
\end{aligned}
$$

Integrating, we arrive at a new functional equation for the quadrilogarithm.

$$
\begin{aligned}
\mathrm{Li}_{4}(1-x)+\mathrm{Li}_{4}\left(1-\frac{1}{x}\right)-\mathrm{Li}_{4}(x)-\mathrm{Li}_{3}(1-x) \ln x \\
=-\frac{1}{24} \ln ^{4} x+\frac{1}{6} \ln ^{3} x \ln (1-x) \\
\quad-\frac{1}{2} \zeta(2) \ln ^{2} x-\zeta(3) \ln x-\zeta(4)+h(1-x)
\end{aligned}
$$

where $1 / 2 \leq x<1$. Solving this for $h(1-x)$ gives us $(1.3)$. 
It is possible to evaluate $h(1 / 2)$ without utilizing (1.3). To do this, we need Ramanujan's entry 10 (i) [5, page 253].

$$
\begin{aligned}
h(1-x)-h\left(1-\frac{1}{x}\right)= & -\frac{1}{24} \ln ^{4} x+\frac{1}{6} \ln ^{3} x \ln (1-x) \\
& +\zeta(3) \ln x+\operatorname{Li}_{3}(x) \ln x+2 \zeta(4)-2 \operatorname{Li}_{4}(x),
\end{aligned}
$$

where $1 / 2 \leq x<1$. For $x=1 / 2$, this yields

$$
\begin{aligned}
h\left(\frac{1}{2}\right)+2 \operatorname{Li}_{4}\left(\frac{1}{2}\right)-h(-1)= & -\frac{1}{24} \ln ^{4} 2+\frac{1}{2} \zeta(2) \ln ^{2} 2 \\
& -\frac{15}{8} \zeta(3) \ln 2+2 \zeta(4) .
\end{aligned}
$$

A formula for $h(-1)$ given by Borwein, Borwein and Girgensohn [7, page 16] can be restated as

$$
2 \mathrm{Li}_{4}\left(\frac{1}{2}\right)-h(-1)=-\frac{1}{12} \ln ^{4} 2+\frac{1}{2} \zeta(2) \ln ^{2} 2-\frac{7}{4} \zeta(3) \ln 2+\frac{15}{8} \zeta(4) .
$$

Subtracting $(1.10)$ from $(1.9)$, we again obtain $h(1 / 2)=(1 / 24) \ln ^{4} 2-$ $(1 / 8) \zeta(3) \ln 2+(1 / 8) \zeta(4)$, which is $(1.4)$.

2. A corrected version. As pointed out in the Frontline article [6], Rediscovering Ramanujan,

Most of the time Ramanujan provided only the results and not the proof.

Thus, Berndt's editing job is doubly difficult. He is interviewed in this article, and one of the things he said especially caught our attention,

I did not count the serious mistakes but it is an extremely small number-maybe five or ten out of over 3,000 results. Considering that Ramanujan did not have any rigorous training, it is really amazing that he made so few mistakes.

The secondary motivation for writing this article is to present what Berndt calls a "corrected version" [5, page 233] of an incorrect formula of Ramanujan. The identity of Ramanujan we address appears in Berndt's Ramanujan's notebooks, Part I. There he explains that, "Unfortunately, this beautiful formula is incorrect." He goes on to say that, 
"We have been unable to find any formula for $G(1)$ which resembles (11.3)" [5, page 257]. Restated slightly, the formula is

$$
\sum_{k=1}^{\infty} \frac{h_{k}}{(2 k)^{3}}=\frac{\pi}{4} \sum_{k=1}^{\infty} \frac{(-1)^{k+1}}{(4 k-3)^{3}}-\frac{\pi}{3 \sqrt{3}} \sum_{k=1}^{\infty} \frac{1}{(2 k-1)^{3}},
$$

where

$$
h_{k}=\sum_{j=1}^{k} \frac{1}{2 j-1} .
$$

Although this is an attractive evaluation, it misses the mark. A thousand terms of

$$
\sum_{k=1}^{\infty} \frac{h_{k}}{(2 k)^{3}}
$$

yields approximately 0.162 . A thousand terms of each sum on the right hand side of the identity yields approximately 0.144 for Ramanujan's expression.

Defining

$$
\lambda(n)=\sum_{k=1}^{\infty} \frac{1}{(2 k-1)^{n}} \quad \text { for } n>1,
$$

(see [1, page 807]), and utilizing Euler's identity found in [4, page 121],

$$
\frac{1}{1}-\frac{1}{2}+\frac{1}{4}-\frac{1}{5}+\frac{1}{7}-\frac{1}{8}+\frac{1}{10}-\frac{1}{11}+\cdots=\frac{\pi}{3 \sqrt{3}}
$$

we can further restate $(2.1)$ as

$$
\sum_{k=1}^{\infty} \frac{h_{k}}{(2 k)^{3}}=\frac{\pi}{4} \sum_{k=1}^{\infty} \frac{(-1)^{k+1}}{(4 k-3)^{3}}-\lambda(3) \sum_{k=1}^{\infty} \frac{1}{(3 k-1)(3 k-2)}
$$

Unexpectedly, we found a formula bearing a strong resemblance to $(2.3)$.

$$
\begin{aligned}
\sum_{k=1}^{\infty} \frac{h_{k}}{(2 k-1)^{3}}+\sum_{k=1}^{\infty} \frac{h_{k}}{(2 k)^{3}}= & \frac{\pi}{6} \sum_{k=1}^{\infty} \frac{(-1)^{k+1}}{(2 k-1)^{3}} \\
& +\lambda(3) \sum_{k=1}^{\infty} \frac{1}{(2 k-0)(2 k-1)}
\end{aligned}
$$


By comparison, a thousand terms of each sum in this identity yields approximately 1.236 for each side. The proof of (2.4) consists of developing the following three identities

$$
\sum_{j=1}^{\infty} \frac{2 k}{(2 j-1)(2 j-1+2 k)}=h_{k}
$$

$$
\sum_{k=1}^{\infty} \sum_{j=1}^{\infty} \frac{1}{(2 k)(2 j-1)^{2}(2 j-1+2 k)}=\frac{1}{4} \zeta(2) \lambda(2)-\sum_{k=1}^{\infty} \frac{h_{k}}{(2 k)^{3}}
$$

$$
\sum_{k=1}^{\infty} \sum_{j=1}^{\infty} \frac{1}{(2 k)(2 j-1)^{2}(2 j-1+2 k)}=-\eta(1) \lambda(3)+\sum_{k=1}^{\infty} \frac{h_{k}}{(2 k-1)^{3}},
$$

where $\eta(n)$ is defined to be

$$
\sum_{k=1}^{\infty} \frac{(-1)^{k+1}}{k^{n}} \text { for } n \geq 1
$$

(see [1, page 807$])$. Note that

$$
\frac{1}{4} \zeta(2) \lambda(2)=\frac{1}{2} \lambda(4)=\frac{2}{3} \beta(1) \beta(3),
$$

where $\beta(n)$ is defined to be

$$
\sum_{k=1}^{\infty} \frac{(-1)^{k+1}}{(2 k-1)^{n}} \quad \text { for } n \geq 1
$$

(see [1, page 807]). The proof of (2.5) is simple and is omitted here. Each double sum identity proof requires a different initial algebraic trick followed by an application of (2.5) at the end of the argument.

First, we have

$$
\begin{aligned}
& \sum_{k=1}^{\infty} \sum_{j=1}^{\infty} \frac{1}{(2 k)(2 j-1)^{2}(2 j-1+2 k)} \\
& =\sum_{k=1}^{\infty} \sum_{j=1}^{\infty} \frac{2 k+(2 j-1)-(2 j-1)}{(2 k)^{2}(2 j-1)^{2}(2 j-1+2 k)}
\end{aligned}
$$




$$
\begin{aligned}
& =\sum_{k=1}^{\infty} \sum_{j=1}^{\infty} \frac{1}{(2 k)^{2}(2 j-1)^{2}} \\
& -\sum_{k=1}^{\infty} \sum_{j=1}^{\infty} \frac{1}{(2 k)^{2}(2 j-1)(2 j-1+2 k)} \\
= & \frac{1}{4} \zeta(2) \lambda(2)-\sum_{k=1}^{\infty} \sum_{j=1}^{\infty} \frac{2 k}{(2 k)^{3}(2 j-1)(2 j-1+2 k)} \\
= & \frac{1}{4} \zeta(2) \lambda(2)-\sum_{k=1}^{\infty} \frac{h_{k}}{(2 k)^{3}} .
\end{aligned}
$$

This completes the proof of (2.6).

Similarly, we have

$$
\begin{aligned}
\sum_{k=1}^{\infty} & \sum_{j=1}^{\infty} \frac{1}{(2 k)(2 j-1)^{2}(2 j-1+2 k)} \\
= & \sum_{k=1}^{\infty} \sum_{j=1}^{\infty} \frac{(2 k-1)(2 j-1)}{(2 k)(2 k-1)(2 j-1)^{3}(2 j-1+2 k)} \\
= & \sum_{k=1}^{\infty} \sum_{j=1}^{\infty} \frac{-(2 j-1+2 k)+(2 k)(2 j)}{(2 k)(2 k-1)(2 j-1)^{3}(2 j-1+2 k)} \\
= & -\sum_{k=1}^{\infty} \sum_{j=1}^{\infty} \frac{1}{(2 k)(2 k-1)(2 j-1)^{3}} \\
& +\sum_{k=1}^{\infty} \sum_{j=1}^{\infty} \frac{2 j}{(2 k-1)(2 j-1)^{3}(2 j-1+2 k)} \\
= & -\eta(1) \lambda(3)+\sum_{j=1}^{\infty} \sum_{k=1}^{\infty} \frac{2 j}{(2 k-1)(2 j-1)^{3}(2 j-1+2 k)} \\
= & -\eta(1) \lambda(3)+\sum_{k=1}^{\infty} \sum_{j=1}^{\infty} \frac{2 k}{(2 k-1)^{3}(2 j-1)(2 j-1+2 k)} \\
= & -\eta(1) \lambda(3)+\sum_{k=1}^{\infty} \frac{h_{k}}{(2 k-1)^{3} .}
\end{aligned}
$$

This completes the proof of (2.7). 
Identities (2.6) and (2.7) give us

$$
\frac{1}{4} \zeta(2) \lambda(2)-\sum_{k=1}^{\infty} \frac{h_{k}}{(2 k)^{3}}=-\eta(1) \lambda(3)+\sum_{k=1}^{\infty} \frac{h_{k}}{(2 k-1)^{3}},
$$

which simplifies to (2.4) since

$$
\frac{1}{4} \zeta(2) \lambda(2)=\frac{2}{3} \beta(1) \beta(3) \quad \text { and } \quad \beta(1)=\frac{\pi}{4} .
$$

This completes the proof of (2.4), our "corrected version" of an incorrect formula of Ramanujan.

3. Intriguing comparisons. The elementary techniques used in this paper can (by lengthy arguments) be used to derive the first and third entries of the following two identity sets. Since

$$
\frac{2}{3} \beta(1) \beta(3)=\frac{1}{2} \lambda(4),
$$

(2.4) can be involved in two additional intriguing comparisons.

$$
\begin{gathered}
\sum_{k=1}^{\infty} \frac{h_{k}}{(2 k)^{2}}=\frac{1}{2} \lambda(3) \\
\sum_{k=1}^{\infty} \frac{h_{k}}{(2 k-1)^{3}}+\sum_{k=1}^{\infty} \frac{h_{k}}{(2 k)^{3}}=\frac{1}{2} \lambda(4)+\eta(1) \lambda(3) \\
\sum_{k=1}^{\infty} \frac{h_{k}}{(2 k)^{4}}=\frac{1}{2} \lambda(5)-\xi(2) \lambda(3) \\
\sum_{k=1}^{\infty} \frac{h_{k}}{(2 k-1)^{2}}=\frac{1}{2} \lambda(3)+\eta(1) \lambda(2) \\
\sum_{k=1}^{\infty} \frac{h_{k}}{(2 k)^{3}}+\sum_{k=1}^{\infty} \frac{h_{k}}{(2 k-1)^{3}}=\frac{1}{2} \lambda(4)+\eta(1) \lambda(3) \\
\sum_{k=1}^{\infty} \frac{h_{k}}{(2 k-1)^{4}}=\frac{1}{2} \lambda(5)+\eta(1) \lambda(4)-\lambda(2) \xi(3),
\end{gathered}
$$


where $\xi(n)$ is defined to be

$$
\sum_{k=1}^{\infty} \frac{1}{(2 k)^{n}} \quad \text { for } n>1
$$

Thus,

$$
\sum_{k=1}^{\infty} \frac{h_{k}}{(2 k-1)^{3}}+\sum_{k=1}^{\infty} \frac{h_{k}}{(2 k)^{3}}
$$

is, in a sense, behaving as one might expect

$$
\sum_{k=1}^{\infty} \frac{h_{k}}{(2 k)^{3}} \quad \text { or } \quad \sum_{k=1}^{\infty} \frac{h_{k}}{(2 k-1)^{3}}
$$

to behave alone.

A quick comment on a "correct" result of Ramanujan is in order here. After constructing a proof of the formula

$$
\sum_{k=1}^{\infty} \frac{h_{k}}{(2 k)(2 k-1)}=\frac{1}{2} \zeta(2)
$$

we were surprised to find a formula of Ramanujan (see [5, page 250]) that can be used in conjunction with the power series for $\frac{1}{2}(\operatorname{arctanh} x)^{2}$ to create a different proof. We did not think it was possible to prove it in any other way, but Ramanujan proved us wrong.

Let us define

$$
{ }_{m} h_{k}=\sum_{j=1}^{k} \frac{1}{(2 j-1)^{m}} \quad \text { for } m \geq 1
$$

For $m=2$, this leads to a third double sum identity that allows us to say something new about $\sum_{k=1}^{\infty} \frac{h_{k}}{(2 k)^{3}}$.

$$
\sum_{k=1}^{\infty} \sum_{j=1}^{\infty} \frac{1}{(2 k)(2 j-1)^{2}(2 j-1+2 k)}=\frac{1}{8} \lambda(4)+\frac{1}{2} \sum_{k=1}^{\infty} \frac{{ }_{2} h_{k}}{(2 k)^{2}} .
$$

Since

$$
\frac{1}{4} \zeta(2) \lambda(2)=\frac{1}{2} \lambda(4)
$$


we can then recall $(2.6)$

$$
\sum_{k=1}^{\infty} \sum_{j=1}^{\infty} \frac{1}{(2 k)(2 j-1)^{2}(2 j-1+2 k)}=\frac{1}{2} \lambda(4)-\sum_{k=1}^{\infty} \frac{h_{k}}{(2 k)^{3}}
$$

and write a new identity reminiscent of

$$
\sum_{k=1}^{\infty} \frac{h_{k}}{k^{2}}=2 \lambda(3)
$$

which is equivalent to $(3.1)$.

$$
\sum_{k=1}^{\infty} \frac{{ }_{2} h_{k}}{k^{2}}+\sum_{k=1}^{\infty} \frac{h_{k}}{k^{3}}=3 \lambda(4)
$$

It can also be shown that

$$
\sum_{k=1}^{\infty} \frac{{ }_{3} h_{k}}{k^{2}}+\sum_{k=1}^{\infty} \frac{{ }_{2} h_{k}}{k^{3}}+\sum_{k=1}^{\infty} \frac{h_{k}}{k^{4}}=4 \lambda(5)
$$

Unlike

$$
\sum_{k=1}^{\infty} \frac{{ }_{2} h_{k}}{k^{2}} \text { and } \sum_{k=1}^{\infty} \frac{h_{k}}{k^{3}}
$$

all three of these sums can be readily evaluated in terms of $\lambda(n)$ and $\zeta(n)$. It is also true that

$$
\sum_{k=1}^{\infty} \frac{{ }_{4} h_{k}}{k^{2}}+\sum_{k=1}^{\infty} \frac{{ }_{3} h_{k}}{k^{3}}+\sum_{k=1}^{\infty} \frac{{ }_{2} h_{k}}{k^{4}}+\sum_{k=1}^{\infty} \frac{h_{k}}{k^{5}}=5 \lambda(6) .
$$

We suspect that $\sum_{k=1}^{\infty} \frac{h_{k}}{k^{5}}$ will share the same fate as $\sum_{k=1}^{\infty} \frac{h_{k}}{k^{3}}$.

A general formula for

$$
\sum_{k=1}^{\infty} \frac{h_{k}}{(2 k)^{2 n}}
$$

exists and can be found in [8, page 682]. Thus, a satisfactory evaluation of

$$
\sum_{k=1}^{\infty} \frac{h_{k}}{(2 k)^{3}}
$$


is an elusive entity; however, it is straightforward to verify that

$$
\sum_{k=1}^{\infty} \frac{H_{k}}{k^{3}}=\frac{1}{2} \zeta^{2}(2)
$$

Using elementary methods that rely on partial sum identities, we also proved the more difficult formula

$$
\sum_{k=1}^{\infty} \frac{H_{k}^{2}}{k^{3}}=\frac{7}{2} \zeta(5)-\zeta(2) \zeta(3) .
$$

Later we learned that this can be derived by more advanced methods (see [3, page 4]). The computational tools employed in these methods are something that Ramanujan did not have available to him. "Computer-aided symbolic manipulation" [2, page 8] and computerized "integer relation detection algorithms" [2, page 3] are now a reality. Ramanujan's path to mathematical truth was not digital, but it must have been delightful.

It should be mentioned that (2.4) is the second of four similar formulas that we proved in basically the same way.

$$
\sum_{k=1}^{\infty} \frac{h_{k}}{(2 k-1)^{2}}-\sum_{k=1}^{\infty} \frac{h_{k}}{(2 k)^{2}}=\eta(1) \lambda(2)
$$

$$
\begin{aligned}
& \sum_{k=1}^{\infty} \frac{h_{k}}{(2 k-1)^{3}}+\sum_{k=1}^{\infty} \frac{h_{k}}{(2 k)^{3}}=\frac{1}{2} \lambda(4)+\eta(1) \lambda(3) \\
& \sum_{k=1}^{\infty} \frac{h_{k}}{(2 k-1)^{4}}-\sum_{k=1}^{\infty} \frac{h_{k}}{(2 k)^{4}}=\eta(1) \lambda(4)+\xi(2) \lambda(3)-\lambda(2) \xi(3) \\
& \sum_{k=1}^{\infty} \frac{h_{k}}{(2 k-1)^{5}}+\sum_{k=1}^{\infty} \frac{h_{k}}{(2 k)^{5}}=\frac{1}{2} \lambda(6)+\eta(1) \lambda(5)-\lambda(3) \xi(3)
\end{aligned}
$$

The last identity can be used to enhance the two identity sets introduced earlier.

$$
\begin{gathered}
\sum_{k=1}^{\infty} \frac{h_{k}}{(2 k)^{2}}=\frac{1}{2} \lambda(3) \\
\sum_{k=1}^{\infty} \frac{h_{k}}{(2 k-1)^{3}}+\sum_{k=1}^{\infty} \frac{h_{k}}{(2 k)^{3}}=\frac{1}{2} \lambda(4)+\eta(1) \lambda(3)
\end{gathered}
$$




$$
\begin{aligned}
\sum_{k=1}^{\infty} \frac{h_{k}}{(2 k)^{4}} & =\frac{1}{2} \lambda(5)-\xi(2) \lambda(3) \\
\sum_{k=1}^{\infty} \frac{h_{k}}{(2 k-1)^{5}}+\sum_{k=1}^{\infty} \frac{h_{k}}{(2 k)^{5}} & =\frac{1}{2} \lambda(6)-\xi(3) \lambda(3)+\eta(1) \lambda(5) \\
\sum_{k=1}^{\infty} \frac{h_{k}}{(2 k-1)^{2}} & =\frac{1}{2} \lambda(3)+\eta(1) \lambda(2) \\
\sum_{k=1}^{\infty} \frac{h_{k}}{(2 k)^{3}}+\sum_{k=1}^{\infty} \frac{h_{k}}{(2 k-1)^{3}} & =\frac{1}{2} \lambda(4)+\eta(1) \lambda(3) \\
\sum_{k=1}^{\infty} \frac{h_{k}}{(2 k-1)^{4}} & =\frac{1}{2} \lambda(5)+\eta(1) \lambda(4)-\lambda(2) \xi(3) \\
\sum_{k=1}^{\infty} \frac{h_{k}}{(2 k)^{5}}+\sum_{k=1}^{\infty} \frac{h_{k}}{(2 k-1)^{5}} & =\frac{1}{2} \lambda(6)+\eta(1) \lambda(5)-\lambda(3) \xi(3) .
\end{aligned}
$$

Now both

$$
\sum_{k=1}^{\infty} \frac{h_{k}}{(2 k-1)^{3}}+\sum_{k=1}^{\infty} \frac{h_{k}}{(2 k)^{3}}
$$

and

$$
\sum_{k=1}^{\infty} \frac{h_{k}}{(2 k-1)^{5}}+\sum_{k=1}^{\infty} \frac{h_{k}}{(2 k)^{5}}
$$

are, in a sense, behaving as one might expect the component sums to behave alone. Identity (3.3) and its successor (3.4) may be as close as one can get to the kind of evaluation Ramanujan was seeking for

$$
\sum_{k=1}^{\infty} \frac{h_{k}}{(2 k)^{3}}
$$

4. A conjecture. Finally, recall from (3.2) that

$$
\frac{1}{2} \sum_{k=1}^{\infty} \frac{{ }_{2} h_{k}}{(2 k)^{2}}+\sum_{k=1}^{\infty} \frac{h_{k}}{(2 k)^{3}}=\frac{3}{8} \lambda(4) .
$$

Clearly, $\sum_{k=1}^{\infty} \frac{2 h_{k}}{(2 k)^{2}}$ shares the same fate as $\sum_{k=1}^{\infty} \frac{h_{k}}{(2 k)^{3}}$, when it comes to being evaluated. With this in mind, we conclude with the following 
conjecture.

$$
\begin{aligned}
\sum_{k=1}^{\infty} \frac{2 n-1}{(2 k)^{2}}= & \frac{1}{4}(2 n-1)(2 n) \lambda(2 n+1) \\
& -\sum_{k=1}^{n-1}(2 k) \lambda(2 k+1) \lambda(2 n-2 k) .
\end{aligned}
$$

Acknowledgments. I wish to thank Professor Paul Ezust of Suffolk University for encouragement.

\section{REFERENCES}

1. M. Abramowitz and I.A. Stegun, eds., Handbook of mathematical functions, Dover, New York, 1965.

2. D.H. Bailey and J.M. Borwein, Experimental mathematics: Recent developments and future outlook, preprint, 1999, available at http://docserver. carma.newcastle.edu.au/250/.

3. D.H. Bailey, J.M. Borwein and R. Girgensohn, Experimental evaluation of Euler sums, preprint, 1993, available at http://docserver.carma. newcastle.edu. au/60/.

4. L. Berggren, J.M. Borwein and P.B. Borwein, Pi: A source book, SpringerVerlag, New York, 1997.

5. B.C. Berndt, Ramanujan's notebooks Part I, Springer-Verlag, New York, 1985.

6. , Rediscovering Ramanujan, Frontline 16, 1999, available at http:// hindu.com/fline/fl1617/16170810.htm.

7. D. Borwein, J.M. Borwein and R. Girgensohn, Explicit evaluation of Euler sums, preprint, 1993, available at http://docserver.carma.newcastle. edu. au/58/.

8. P.F. Jordan, Infinite sums of Psi functions, Bull. Amer. Math. Soc. 79 (1973), 681-683. 1981.

9. L. Lewin, Polylogarithms and associated functions, North Holland, New York,

10. , ed., Structural properties of polylogarithms, American Mathematical Society, Providence, RI, 1991.

Suffolk University, Boston, MA 02108

Email address: knopp010@verizon.net 